\title{
Improving Vehicle Detection Accuracy Based on Vehicle Shadow and Superposition Elimination
}

\author{
Hongjin Zhu ${ }^{*},{ }^{1}$ Honghui Fan ${ }^{1}$, Feiyue Ye ${ }^{1,2}$ and Xiaorong Zhao ${ }^{1}$ \\ ${ }^{I}$ School of Computer Engineering, Jiangsu University of Technology, Changzhou, 213001, China \\ ${ }^{2}$ Key Laboratory of Cloud Computing \& Intelligent Information, Changzhou, 213001, China
}

\begin{abstract}
Vehicle shadow and superposition have a great influence on the accuracy of vehicles detection in traffic video. Many background models have been proposed and improved to deal with detection moving object. This paper presented a method which improves Gaussian mixture model to get adaptive background. The HSV color space was used to eliminate vehicle shadow, it was based on a computational colour space that makes use of our shadow model. Vehicle superposition elimination was discussed based on vehicle contour dilation and erosion method. Experiments were performed to verify that the proposed technique is effective for vehicle detection based traffic surveillance systems. Detection results showed that our approach was robust to widely different background and illuminations.
\end{abstract}

Keywords: Dilation and erosion, gaussian mixture, HSV color space, shadow and superposition, vehicle detection.

\section{INTRODUCTION}

Moving object detection in image sequences is fundamental in application areas such as automated visual surveillance, content-based video compression, and automatic traffic monitoring [1]. Automated video surveillance has emerged as an important research topic in the computer vision community. There are many reports on vehicle detection and tracking in traffic video, purpose is to build an intelligent transportation system for solving the problems of road traffic congestion and traffic accidents in urban highway main road [2,3]. Intelligent transportation system has an important role in command and control system of traffic. Many new research topics in image processing have been proposed, and there has also some problems need to be resolved in intelligent transportation systems, such as creating a stable algorithm for adapting to the various light conditions, and high performance computing ability, so as to achieve the requirements of real-time intelligent traffic monitoring system [4]. There have been many research results in this field, and there are some products have been applied to the road traffic [5], but these traffic monitoring systems based on computer vision, they have significantly improved and enhanced space on the performance and functionality [6].

Many research reports discussed the potential and commercial of a machine vision system for traffic monitoring and control [7]. To accelerate the intelligent transportation system processes, many scientists have made efforts to utilize image analysis to extract useful information, Nakanlshi and Ishi [8] presented a method for extracting moving vehicle image from the image sequence based on image analysis of spatio-temporal. Elgammal and Haritaoglu discussed the linear process and probability distribution can be characterized background changes in their research reports $[9,10]$. Kalman filter was used to track the changes of every pixel in background illumination by Koller et al., and they applied a selective update scheme for possible background value into estimate of background $[11,12]$. This often makes the segmentation of moving vehicle parts from the background parts in traffic images. In addition, some researched the effect of light illumination changes of vehicle detection. In the system described by Agostino, problems of vehicle shadow and strong light changes in vehicle detection had not been solved. In the reporter [13], foreground was separated from the shadow by intensity dynamic and integration of gradient. The real system should have environmental adaptation, it can work in different light conditions, but there are some problems in practical applications, one of them is the vehicle separation from overlapping and shadow of vehicle [14]. However, accurate detection in intelligent transportation system is difficult due to the potential variability such as shadows and superposition of vehicle [15].

In this paper, an approach of moving vehicle detection is proposed based on vehicle shadow and superposition elimination for automatic traffic monitoring. The moving vehicle detection algorithm was developed to elimination the effects of light using Gaussian mixture model and morphology corrosion expansion. Experimental results showed that the proposed approach effectively extracts vehicle contour features in traffic image sequences, and significantly improves the vehicle detection accuracy.

\section{IMPROVED GAUSSIAN MIXTURE MODEL}

Gaussian mixture model (GMM) parameter of the probability density function is expressed as a weighted sum of the density of gaussian components. GMMs are generally 
used as parametric models of probability distribution of continuous measurements or features, such as tracking of an object in video. Although each pixel based on gaussian mixture model with $K$ ( $K$ generally take $3-5)$ gaussian distribution to represent the characteristics of an image pixel. Each state of $K$ represented by a gaussian function, part of the state represents the background pixel value, the rest represents the movement prospects pixel value. If each pixel color values expressed in variable $X_{t}$, the probability density function is available $K$ gaussian functions as equation (1).

$f\left(X_{t}=x\right)=\sum_{i=1}^{k} \omega_{i, t} * \eta\left(x, u_{i t}, \sum_{i, t}\right)$.

$\eta\left(x, u_{i, t}, \sum_{i, t}\right)$ is $i_{t h}$ gaussian distribution in $t$ time, $u_{i, t}$ is mean value, $\sum_{i, t}$ is covariance matrix, the $k t h$ covariance matrix is calculated as follows.

$\sum_{k}=\sigma_{k}^{2} I$.

$\omega_{i, t}$ is weight of the $i_{t h}$ gaussian distribution, and $\sum_{i=1}^{k} \omega_{i, t}=1$. In here,

$$
\eta\left(x, u_{i, t}, \sum_{i, t}\right)=\frac{1}{(2 \pi)^{\frac{n}{2}} \sqrt{\left|\sum_{i, t}\right|}} \exp \left[-\frac{1}{2}\left(x-u_{i, t}\right)^{T} \sum^{-1} x-u_{i, t}\right] .
$$

$n$ is the dimension of $X_{t}$. To improve system operation speed, based on gaussian mixture model to simulate the gray image background modeling, so when $n=1$, the computing speed is fastest. For a new time $N$ pixels $x_{N}$, according to the traversal sequence of the gaussian background model, check whether it matches a Gaussian model. If the gaussian model satisfying condition $\left|x_{N}-u_{k}\right| \leq 2.5 \sigma_{k}$, it is considered to be matched. Update strategy of the GMM is our system as follows.

$\omega_{k}^{\prime}=(1-\alpha) \omega_{k}+\alpha p$

$u_{k}^{\prime}=(1-\alpha) u_{k}+\rho x_{N}$

$\sum_{k}^{\prime}=(1-\alpha) \sum_{k}+\rho\left(x_{N}-u_{k}^{\prime}\right)\left(x_{N}-u_{k}^{\prime}\right)^{T}$

$\rho=\alpha \eta\left(x_{N}, u_{k}, \sum_{K}\right)$.

$T$ is background resolution threshold, $\alpha$ is learning rate, it determines the model updating learning the proportion of new pixels, and parameter $p$ is explained as equation (5).

$p= \begin{cases}1 & \text { (pixel value matching model) } \\ 0 & \text { (otherwise) }\end{cases}$

Although GMM can detect moving targets, but it also has some shortcomings and deficiencies. The disadvantages include object contour unclear of foreground, sensitivity to light and easily affected by noise. To overcome the imperfections in image processing, an improvement method was proposed to detect moving object using GMM and Small Univalue Segment Assimilating Nucleus (SUSAN) edge detector. The USAN region of one pixel is denoted equation (6).

$m\left(r_{o}\right)=\sum c\left(r, r_{o}\right)$

where $r_{o}$ is core pixel position (kernel), $r$ is others inner pixels position, $c\left(r_{o}\right)$ is a circle template on the centre $r_{o}$, $c\left(r, r_{o}\right)$ is a judgment function of the pixels which belong to the USAN region or not, it is denoted as follows.

$c\left(r, r_{o}\right)=\left\{\begin{array}{l}1 \text { if }\left|I(r)-I\left(r_{o}\right)\right| \leq t \\ 0 \text { if }\left|I(r)-I\left(r_{o}\right)\right|>t\end{array}\right.$

$I\left(r_{o}\right)$ is the gray value of the template center (kernel), $I(r)$ is other pixel gray value inside the template, $t$ is the threshold of gray value. In practical application, based on equation (8), we can get more stable and efficient comparison function. In practice, a more stable and efficient comparative function is adopted.

$c\left(r, r_{o}\right)=\exp \left(-\left(\frac{I(r)-I\left(r_{o}\right)}{t}\right)^{a}\right)$.

The combination of GMM and SUSAN can effectively restrain the shadow and get more precise foreground object. On the other hand, SUSAN is a feature point acquisition method based on gray-scale, and is applied to the image edge and corner detection, so it has strong adaptability to light, it can effectively solve the second problem and improve stability to reduce the influence of the noise.

\section{VEHICLE SHADOW AND SUPERPOSITION ELIMINATION}

In vehicle detection system, the shadow should be removed for improving the accuracy of intelligent transportation systems. The vehicle shadow model is considered in our vehicle detection system. The shadow of a vehicle is decomposed into the length and width in both directions. In our system, chromatic colour space representation method is utilized to reduce susceptibility, so the colour spaces could separate illumination and chromatic. Maintaining a chromatic model of the brightness can find the chroma is constant, the brightness of the small area is an area shadow in traffic image. Shadow areas can be separated by Equation (9), and $M_{1}$ is the binary of the shadow mask renewed after processing.

$M_{1}(x, y)=\left\{\begin{array}{c}1: \text { if } T_{\text {min }}^{H} \leq H(x, y) \leq T_{\text {max }}^{H} \\ \wedge\left(T_{\text {min }}^{S} \leq S(x, y) \leq T_{\text {max }}^{S}\right) \\ \wedge\left(T_{\text {min }}^{V} \leq V(x, y) \leq T_{\max }^{V}\right) . \\ \\ 0 \text { :otherwise }\end{array}\right.$

$M_{2}(x, y)=\left\{\begin{array}{l}1: \text { if }(b(x, y) \geq r(x, y) \\ \wedge(b(x, y) \geq g(x, y) \\ \wedge M_{1} \\ 0 \text { :otherwise }\end{array}\right.$. 


$$
\begin{aligned}
& H=\left\{\begin{array}{l}
60 \times \frac{B-R}{M A X-M I N}+0 \text { if } M A X=R \\
60 \times \frac{B-R}{M A X-M I N}+120 \text { if } M A X=G \\
60 \times \frac{R-G}{M A X-M I N}+240 \text { if } M A X=B \\
H+360 \text { if } H<0
\end{array}\right. \\
& S=\frac{M A X-M I N}{M A X} \times 100 \\
& V=M A X \times \frac{100}{255} .
\end{aligned}
$$

The scope of HSV value specifies the shadow areas, and $\mathrm{T}$ is the threshold. The largest components of blue component are used as an observation in this method. But the brightness is very weak for blue, so it was thought that blue becomes a lot at a part in dark part and shadow of RBG colour system. The second binary $M_{2}$ is obtained by Equation 10 .

Because HSV value display Linux image processing software XV which were used in this study according to the following Equation 11. and,

$$
M A X=\max (R, G, B), M I N=\min (R, G, B) .
$$

Shadow does not have the color characteristics, and therefore will have a transparent color feature moving pixels within the region as vehicle extraction areas. Hatched region can not have a high luminance value, on the contrary, the luminance of the pixel are low, in the RGB color space, the $R$ values of the color characteristic of the large ratio of the pixel value of $G$ and $B$ as the vehicle characteristics retained. As showed in Fig. (1), extract high brightness for a vehicle roof section (yellow), the lower the brightness of the tire section (blue), red brake lights for observable characteristics (red). Based on the detected characteristics, we can get the vehicle area of long, wide, high parameter, matching the vehicle model, so as to get more accurate vehicle area. Based on the definition of the shadow color information model, the shadow of the car was eliminated, and some of the body parts of the information contained shadow color characteristics were eliminated too. Therefore, it is necessary for the recovery process for vehicle field after the elimination of shadows. Features based on color information of the vehicle, vehicle interference from shadow to extract the vehicle area. However, due to the different body colors, there are a few in the shadows most of the vehicle body are eliminating errors eliminated.

Fig. (1a) is the pixel having a characteristic color of the vehicle extracted from the moving object area. It is possible to obtain the parameters of the height and width of the vehicle does not include a shadow region, equation (12) is the expression of feature points.

$$
\begin{aligned}
f_{p}(x, y) & =(V(x, y) \geq 70) \\
& \vee(V(x, y) \leq 20) \\
& \vee(r(x, y) \geq g, b(x, y)+30 \%)
\end{aligned}
$$

(a) Original Image

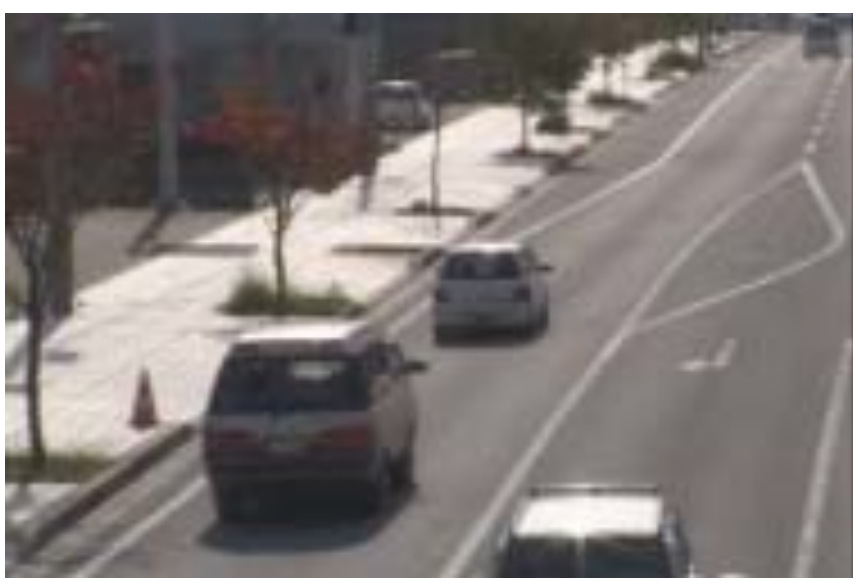

(b) Feature Image

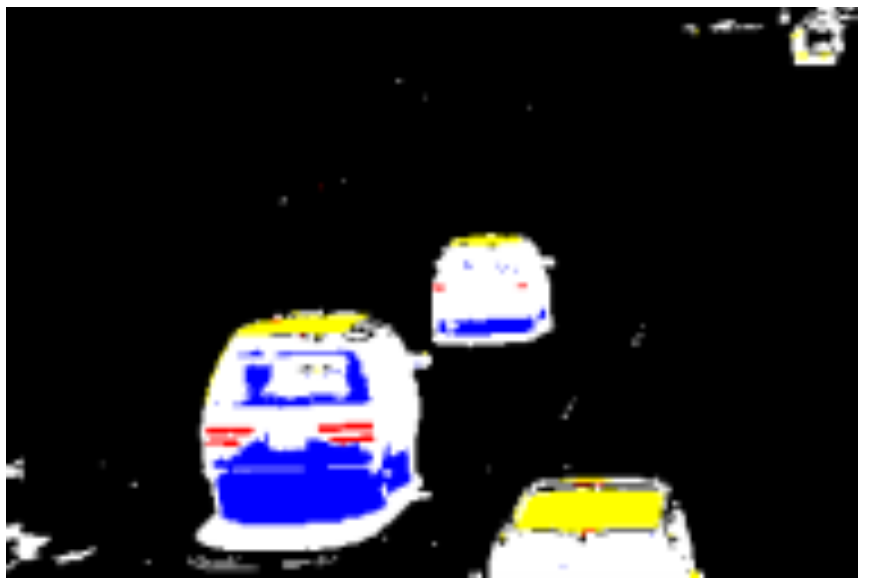

Fig. (1). Feature extraction image.

Morphology computation is a theory and technique which used to analyze and process the geometrical structures. Because of vehicle shadow and occlusion influence in traffic video, intelligent transportation systems cannot accurately detect the number or information of vehicles. Image binary treatment can effectively reduce the influence of other factors, though binary image may miss detail information. Dilation and erosion computation processing could remove shadow and occlusion by accounting for the structure and form of traffic image, and these techniques have been extended to gray scale images.

Dilation and erosion are dual operations in that they have opposite effects, and the results of dilation and erosion are influenced the shape and size of the structuring element. Fig. (2) showed the vehicle area detection result based on dilation and erosion.

\section{EXPERIMENTAL RESULTS}

Fig. (3a) is traffic image of traffic sequences, as the experimental images. Fig. $(\mathbf{3 b}, \mathbf{c})$ shows the processing results of experimental image, in particular joined restrictions $b \geq r, g$ processing output. The shadow removal result could be got used to the color information, the result extracted asphalt part of dilation processing remains. 


\section{(a) Original Image}

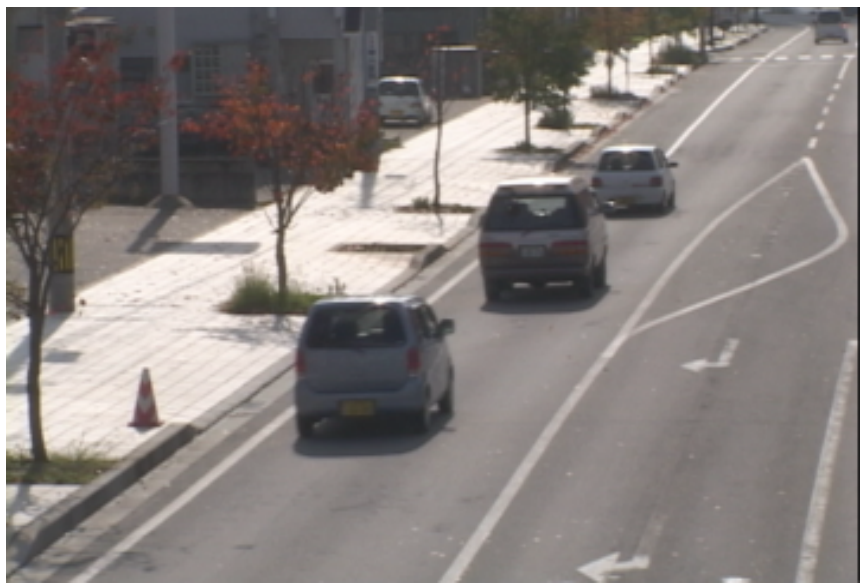

(b) Vehicle Superposition

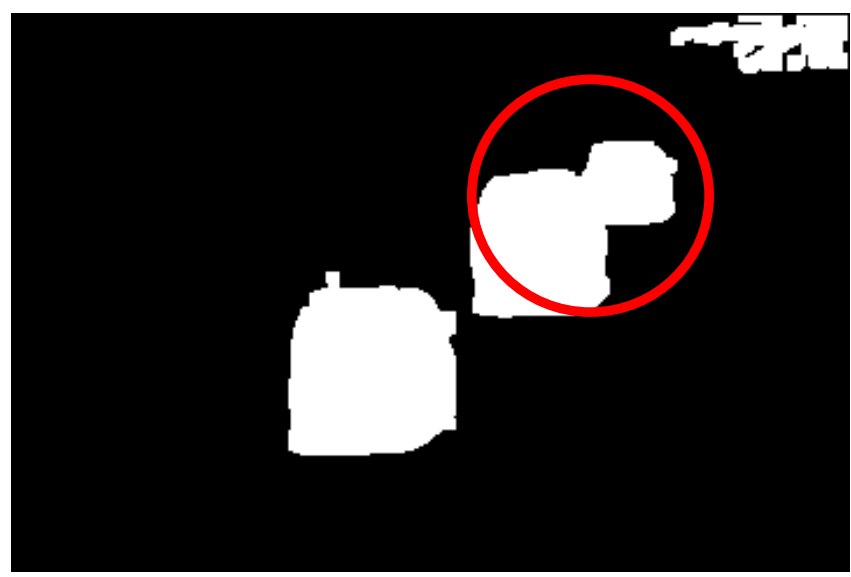

(c) Superposition Elimination

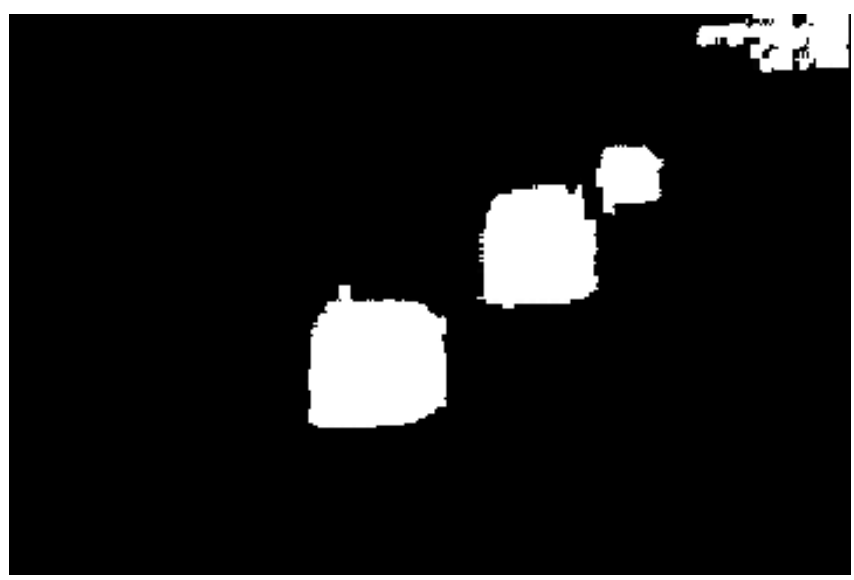

Fig. (2). Vehicle superposition elimination.

Movement vehicle territory was picked based twice dilation processing, the vehicle feature point observed in the movement area was showed in Fig. (4). Vehicle shadow region was eliminated by the model with the movement area part of the expanded outside by dilation processing.

Vehicle detection result was showed in Fig. (5), the vehicle shadow could be separated by the vehicle model. For different images in diverse shadow model, the vehicle could be detected, and the vehicle shadow can be excluded. To avoid doubt vehicle detection, the system was adapted for large vehicles. Likewise, the moving direction of the vehicle had no effect on the detection accuracy.

(a) Original Image

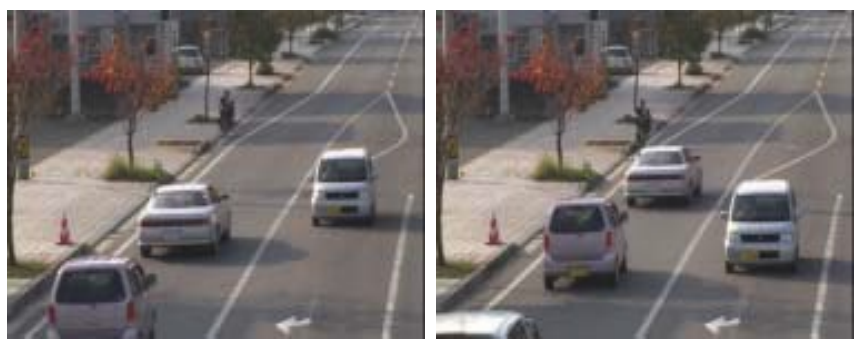

(b) Shadow Area by HSV

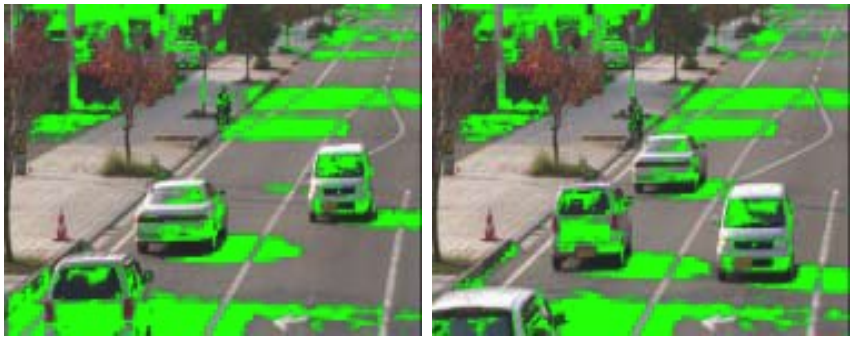

(c) Shadow Detection

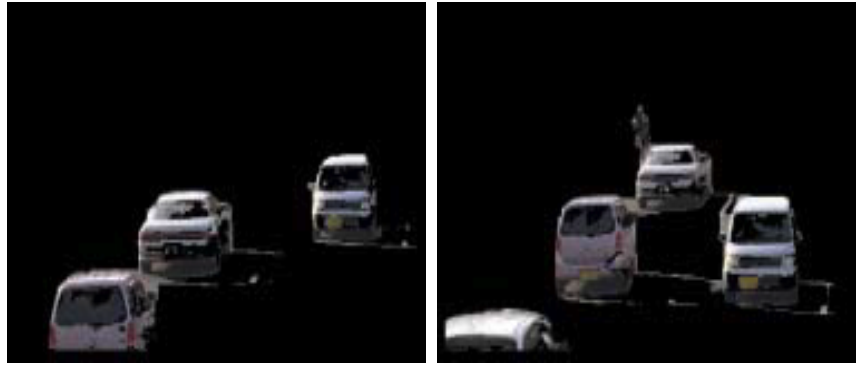

Fig. (3). Vehicle shadow detection based HSV.

Based on HSV color space, the definition of the shadow area vehicle extract, and the movement vehicle could be extracted, nor affected by shading between vehicles. However, due to the color of the shadow as the sun sunshine conditions often change. In order to define the color data for the shadow, the parameters have to continue input. defined the color data of the same color of the vehicle part have been eliminated, especially in order to extract the vehicle field, closing processing and color change increases the amount of calculation. In order to solve this problem, if using the characteristics of the shadow in the outdoor environment, to implement the shadow elimination processing, for changes in the environment, can automatically search the shadow image parameter data, the development of automatic corresponding procedure is necessary condition.

\section{CONCLUSION}

Based on HSV color space color data, using shadow features to eliminate the vehicle shadow, the effectiveness of the vehicle shadow was verified. At the same time, two dimensional simple vehicle models of vehicle model for shadow separation processing technique also be discussed. Using characteristics of shadow, eliminate the shadow area is 
(a) Original Image
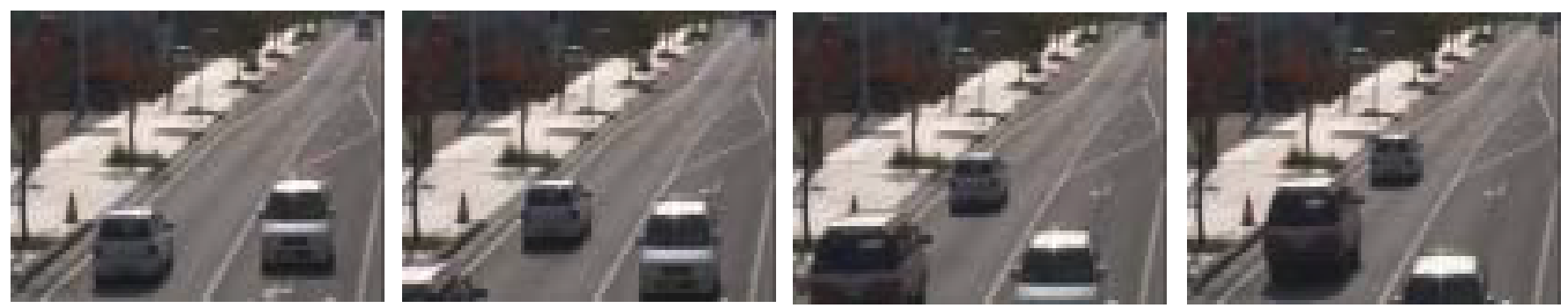

(b) Moving Area Extraction
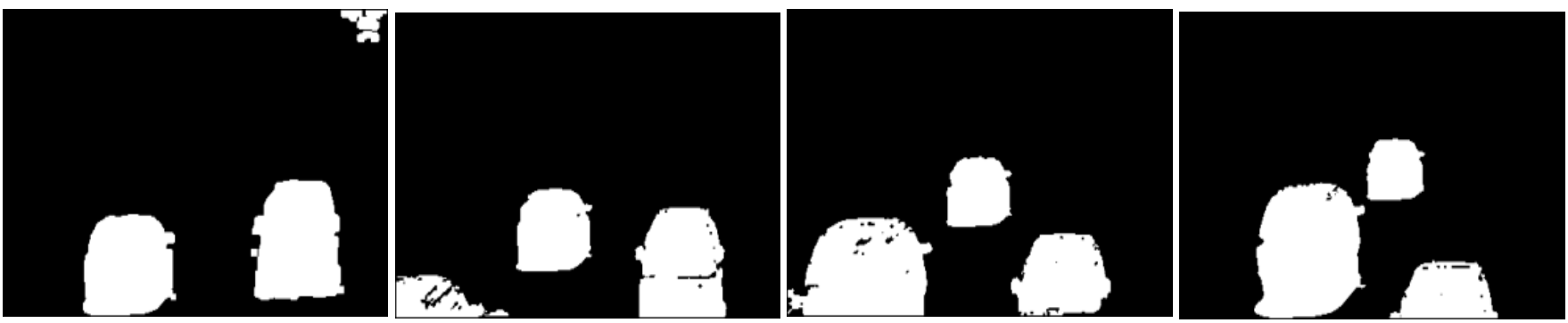

(c) Extraction of Feature Points
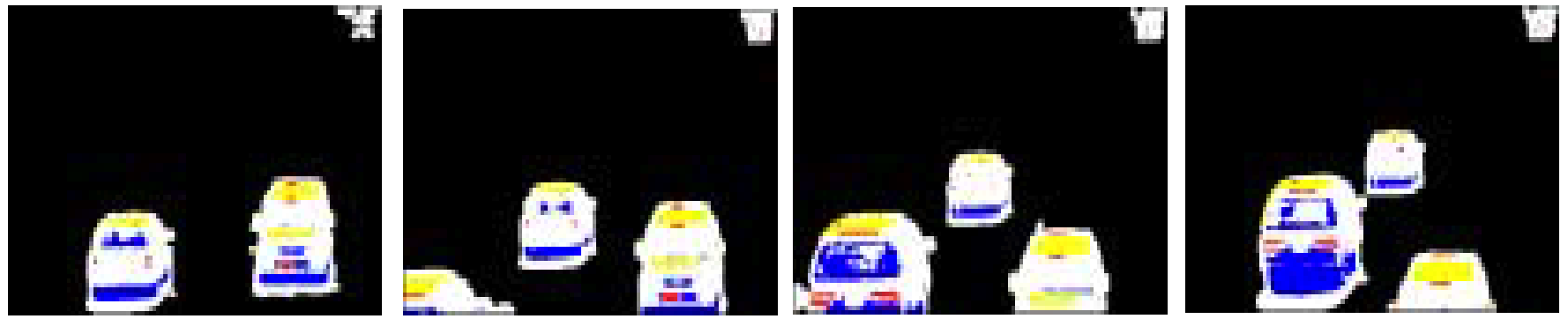

Fig. (4). Vehicle feature point observed in movement area.
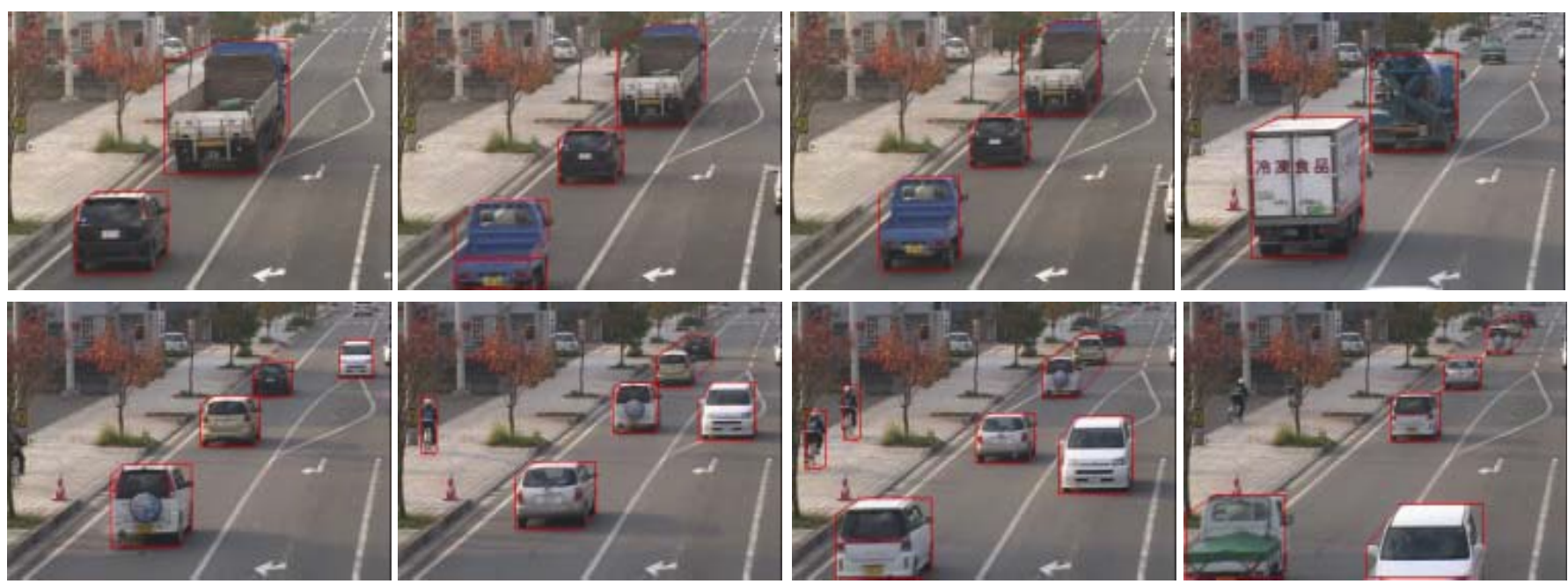

Fig. (5). Vehicle detection result.

easy, because environmental change the parameters of the shadow detection are necessary to solve the problem. The vehicle model separation technique is effective. In addition, because there is not any prior treatment, the algorithm is simple, to extract significantly enhance processing speed. Other environmental condition detection and superposition are considered in the next research.

\section{CONFLICT OF INTEREST}

The authors confirm that this article content has no conflict of interest.

\section{ACKNOWLEDGEMENTS}

The authors are very thankful to Koga LAB from Yamagata University of Japan for providing experimental 
data. This work was supported by Natural Science Fund of Jiangsu Province (BK20130235), Natural Science Fund of Changzhou (CJ20140049), China National Natural Science Fund of China (61302124 and 61472166), Natural Science Foundation of the Higher Education Institutions of Jiangsu Province (13KJB520006), and Program of six talent tops of Jiangsu Province (DZXX-031), Scientific Research Foundation of Jiangsu University of Technology (KYY14020).

\section{REFERENCES}

[1] Y. Wang, "Joint random field model for all-weather moving vehicle detection," IEEE Transactions on Image Processing, vol. 19, no. 9, pp. 2491-2501, 2010

[2] H. Zeng, X. Wang, C. Cai, and J. Chen, "Fast multiview video coding using adaptive prediction structure and hierarchical mode decision," IEEE Transactions on Circuits and Systems for Video Technology, vol. 24, no. 9, pp. 1566-1578, 2014.

[3] J. D. Lee, and K. F. Huang, "A warning system for obstacle detection at vehicle lateral blind spot area," In: Proceedings of the 2013 7th Asia Modelling Symposium, Hong Kong, 2013, pp. 154159.

[4] S. Sivaraman, and M. M. Trivedi, "Active learning for on-road vehicle detection: A comparative study," Machine Vision and Applications, vol. 25, no. 3, pp. 599-611, 2014.

[5] Y. C. Kuo, N. S. Pai, and Y. F. Li, "Vision-based vehicle detection for a driver assistance system," Computers and Mathematics with Applications, vol. 61, no. 8, pp. 2096-2100, 2011.

[6] T. Nakanishi, and K. Ishii, "Automatic vehicle image extraction based on spatio-temporal image analysis," In: Proceedings of LTH $I C P R, 1992$, pp. 500-504.
[7] E. Ahmed, D. Ramani, H. David, and D. Larry, "Background and foreground modeling using nonparametric kernel density estimation for visual surveillance," Proceedings of the IEEE, vol. 90, no. 7, pp. 1151-1163, 2002.

[8] H. Ismail, H. David, and D. Larry, "W4: Real-time surveillance of people and their activities," Pattern Analysis and Machine Intelligence, vol. 22, no. 8, pp. 809-830, 2000.

[9] D. Koller, J. Weber, T. Huang, J. Malik, G. Ogasawara, B. Rao, and S. Russell, "Towards robust automatic traffic scene analysis in realtime. Pattern Recognition," Conference A: Computer Vision \& AMP; Image Processing, Proceedings of the 12th IAPR International Conference on. IEEE, vol. 1, 1994, pp. 126-131.

[10] Y. Wang, K. F. Loe, and J. K. Wu, "A dynamic conditional random field model for foreground and shadow segmentation," IEEE Transactions on Pattern Analysis and Machine Intelligence, vol. 28, no. 2, pp. 279-289, 2006.

[11] T. Nawaz, F. Poiesi, and A. Cavallaro, "Measures of effective video tracking," IEEE Transactions on Image Processing, vol. 23, no.1, pp. 376-388, 2013.

[12] M. Betke, E. Haritaoglu, and L. S. Davis, "Real-time multiple vehicle detection and tracking from a moving vehicle," Machine Vision and Applications, vol. 12, no. 2, pp. 69-83, 2000.

[13] H. Fan, and H. Zhu, "Separation and extraction of moving vehicles in traffic images based on horizontal roof edges," International Review on Computers and Software, vol. 7, no. 3, pp. 1347-1351, 2012.

[14] A. Jazayeri, H. Cai, J. Y. Zheng, and M. Tuceryan, "Vehicle detection and tracking in car video based on motion model," IEEE Transactions on Intelligent Transportation Systems, vol. 12, no. 2, pp. 583-595, 2011

[15] M. S. Javadi, M. A. Hannan, S. A. Samad, and A. Hussain, "A robust vision-based lane boundaries etection approach for intelligent vehicles," Information Technology Journal, vol. 11, no. 9, pp. 1184-1192, 2012. 